\title{
DISCRETE PHENOMENA IN UNIQUENESS IN THE CAUCHY PROBLEM ${ }^{1}$
}

\author{
FRANÇOIS TREVES
}

ABSTRACT. The concatenation method, recently applied to the study of solvability and hypoellipticity of linear partial differential equations with double characteristics, is used to show that uniqueness in the Cauchy problem, for certain degenerate hyperbolic equations, depends on whether the lower order terms (precisely, the subprincipal part) assume or not certain values belonging to a sequence of complex numbers.

1. Introduction. Recent work [4], [3], [5], [6], [9], [1], [2] on the local solvability and hypoellipticity of pseudodifferential equations with double characteristics has revealed a number of discrete phenomena, similar, and closely related, to those encountered in the study of asymptotic solutions of second-order (linear) O.D.E.'s. In conformity with the argument in [8] one should expect parallel developments in the theory of uniqueness in the Cauchy problem. This note seeks to confirm this fact by studying the example of the following operator (in $\mathbf{R}^{2}$ ):

$$
\mathbf{P}=\partial_{x}^{2}-x^{2} \partial_{y}^{2}-\lambda \partial_{y}
$$

where $\lambda$ is a real parameter. We shall be interested in uniqueness across the line $y=0$; it should be noted that this line is everywhere noncharacteristic, except at the origin-where it is doubly characteristic. We shall prove the following two theorems:

Theorem I. Suppose that $\lambda$ is not equal to an odd integer $>0$. Let $\Omega$ be an open subset of the plane, $F$ a closed subset of $\Omega$ whose intersection with the closed half-space $y \geq 0$ is a compact set $K$. There is an integer $m \geq 2$, depending solely on $\lambda$, and an open neighborhood $U$ of $K$ such that

Received by the editors June 12, 1973.

AMS (MOS) subject classifications (1970). Primary 35L15, 35 A05.

Key words and phrases. Cauchy problem, concatenation, uniqueness, degenerate hyperbolic.

1 Work partly supported by NSF Grant 27671. 
every $\mathcal{C}^{m}$ function $u$ with support in $F$, which satisfies $\mathbf{P} u=0$ in $\Omega$, vanishes in $U .^{2}$

Theorem II. Suppose that $\lambda=2 p+1, p \in \mathbf{Z}_{+}$. Then there exists a $C^{\infty}$ function $u$ in $\mathbf{R}^{2}$, whose support is exactly the closed set defined by $y+$ $x^{2} / 2 \leq 0$ and which satisfies $\mathbf{P} u=0$ in $\mathbf{R}^{2}$.

Thus the function $u$ in the conclusion of Theorem II has a support which intersects the upper half-space $y \geq 0$ exactly at the origin; this is contrary to what happens when $\lambda$ is not an odd integer.

The reader will also note that, when $\lambda$ is a positive odd integer, there is no uniqueness in the Cauchy problem across the $x$-axis for functions supported in the lower half-plane (according to Theorem II), whereas there is uniqueness across that same straight line for functions supported in the upper half-plane (according to Theorem I). This is an instance of one-sided uniqueness.

The results and methods described here are a particular case of a general theory, paralleling that developed for local solvability and hypoellipticity. But the present note is self-contained (except for the use of Carleman's method in the uniqueness in the Cauchy problem). We prove Theorems I and II by the method of concatenations (introduced in [8]).

2. Proof of Theorem I. Consider the differential operator

$$
\mathbf{P}^{\#}=e^{\tau y} \mathbf{P} e^{-\tau y}=\partial_{x}^{2}-x^{2}\left(\partial_{y}-\tau\right)^{2}-\lambda\left(\partial_{y}-\tau\right) .
$$

Let us denote by $($,$) and \|\|$, respectively, the inner product and the norm in $L_{x, y}^{2}$. We have, for an arbitrary $v \in C_{c}^{\infty}\left(\mathbf{R}^{2}\right)$,

$$
\operatorname{Re}\left(\mathbf{P}^{\#} v, v_{y}\right)=2 r\left\|x v_{y}\right\|^{2}-\lambda\left\|v_{y}\right\|^{2} \text {. }
$$

Let us suppose that the support of $v$ is contained in a fixed bounded subset of $\mathbf{R}^{2}$ (large enough, so that its interior contains $K$ ). If $\lambda<0$, we derive from (2.1):

2 It is quite likely that the integer $m$ can be chosen independently of $\lambda$, in fact equal to two, and that this could have been established-at the price of some extra effort. But our purpose here is merely to show the role of a sequence of exceptional values of the parameter $\lambda$ in the question of uniqueness in the Cauchy problem, and such extra effort has not seemed warranted.

The relation between $m$ and $\lambda$ which comes out of the proof of Theorem $I$ can easily be made precise: we can take for $m$ the smallest integer (not less than 2) such that $\lambda-2(m-2)<0$. It will also appear, in the proof, that the neighborhood $U$ can be chosen independently of $\lambda$. 


$$
\|v\| \leq C|\lambda|^{-1}\left\|\mathbf{P}^{\#} v\right\|
$$

Let us then set $v=e^{\tau y} w$. We derive from (2.2):

$$
\iint e^{2 \tau y}|w|^{2} d x d y \leq C^{2}|\lambda|^{-2} \iint e^{2 \tau y}|\mathbf{P} w|^{2} d x d y .
$$

We apply Theorem 2.3 of [7] and derive that there is an open neighborhood $U$ of $K$ such that every $C^{2}$ function $u$ satisfying $\mathbf{P} u=0$ in $\Omega$ such that supp $u \subset F$ must vanish in $U$. This proves Theorem I under the additional assumption that $\lambda<0$. Let us emphasize the fact (obvious on inspection of the proof of Theorem 2.3 of [7]) that the neighborhood $U$ can be chosen independently of $\lambda<0$. Possibly after some decreasing of $U$ we may assume that each one of its connected components intersects $\Omega \backslash F$. We shall use this property below.

We shall now suppose $\lambda \geq 0$ but different from any odd integer. We shall use the method of concatenations, introduced in [8]. We may write

$$
\mathbf{P}=X Y-\mu \partial_{i}
$$

where:

$$
X=\partial_{x}+x \partial_{y}, \quad Y=\partial_{x}-x \partial_{y}, \quad \mu=\lambda-1 .
$$

We form a sequence $\mathbf{P}^{j}(j=0,1, \ldots)$ of differential operators as follows:

$$
\mathbf{P}^{0}=\mathbf{P}, \quad \mathbf{P}^{j}=\mathbf{P}-j[X, Y]=X Y-(\mu-2 j) \partial_{y^{*}}
$$

We have the concatenation relations:

$$
Y \mathbf{P}^{j}=\mathbf{P}^{j+1} Y .
$$

Suppose that we have proved the following (for some $m \geq 2$ ):

$$
\begin{aligned}
& \text { Every } u \in C^{m}(\Omega) \text { which satisfies } \mathbf{P}^{j+1} u=0 \text { in } \Omega, \\
& \text { such that supp } u C F, \text { must vanish in } U .
\end{aligned}
$$

We are going to show that (2.7) is still true if we substitute $j$ for $j+1$ and $m+1$ for $m$. Let $u \in C^{m+1}(\Omega)$, supp $u \subset F, \mathbf{P}^{j} u=0$ in $\Omega$. By (2.6) and (2.7) we derive that $Y u=0$ in $U$. But then $X Y u=0$ in $U$ and therefore $(\mu-2 j) \partial_{y} u=X Y u$ $-\mathbf{P}^{j} u=0$ in $U$. Since $\lambda$ is not an odd integer, $\mu-2 j \neq 0$, and therefore $\partial_{y} u=0$ in $U$; since $Y u=0$ in $U$, we see that $\partial_{x} u=0$ in $U$, hence $u$ is locally constant in $U$. Since every connected component of $U$ intersects the complement of supp $u$, we have $u=0$ in $U$.

As soon as $2(j+1)>\mu$ we know from the result for $\lambda<0$ that (2.7) holds. By descending induction on $j$ we see that, for a suitably large 
integer $m$ (depending on $\lambda$ ), (2.7) is true with $\mathbf{P}$ substituted for $\mathbf{P}^{j+1}$. Q.E.D.

3. Proof of Theorem II. We assume $\lambda=2 p+1, p \in \mathbf{Z}_{+}$. With the notation (2.4)-(2.5) this means that $\mu=2 p$. We use the same concatenation $\mathbf{P}^{j}(j=0,1, \ldots)$ as in $\$ 2$ but instead of (2.6) the other concatenation relations:

$$
X \mathbf{P}^{j+1}=\mathbf{P}^{j} X
$$

For $j=p-1, \mathbf{P}^{j+1}=X Y$, hence:

$$
X^{p+1} Y=\mathbf{P} X^{p}
$$

Let $h(t)$ be a $C^{\infty}$ function on the real line, vanishing identically for $t>0$. We have, trivially,

$$
Y\left[h\left(y+x^{2} / 2\right)\right]=0 \text { in } \mathbf{R}^{2} .
$$

Consequently, $u(x, y)=X^{p}\left[h\left(y+x^{2} / 2\right)\right]$ is a $C^{\infty}$ function in $\mathbf{R}^{2}$, satisfying $\mathbf{P} u=0$ in $\mathbf{R}^{2}$ and whose support is contained in the parabolic region

$$
y+x^{2} / 2 \leq 0
$$

If we perform the change of variables $x^{\prime}=x, y^{\prime}=y-x^{2} / 2$, the vector field $X$ becomes $\partial_{x}$ and

$$
u(x, y)=\partial_{x^{\prime}}^{p}\left[h\left(y^{\prime}+x^{\prime 2}\right)\right] .
$$

It is then obvious that we may choose $h(t)$ in such a way as to insure that the support of $u$ is exactly equal to the closed set (3.4). For instance, we may take

$$
h(t)=\exp (1 / t) \text { for } t<0, \quad h(t)=0 \text { for } t>0 \text {. }
$$

\section{REFERENCES}

1. L. Boutet de Monvel and F. Treves, On a class of pseudodifferential operators with double characteristics, Invent. Math. 24 (1974), 1-34.

2. - On a class of systems of pseudodifferential equations with double characteristics, Comm. Pure Appl. Math. 27 (1974), 59-89,

3. A. Gilioli and F. Treves, An example in the solvability theory of linear $P D E$ 's, Amer. J. Math. (to appear).

4. V. V. Grušin, On a class of elliptic pseudodifferential operators degenerate on a submanifold, Mat. Sb. 84 (126) (1971), 163-195 = Math. USSR Sb. 13 (1971), 155-186. MR $44 \# 860$.

5. J. Sjostrand, Une classe d'operateurs pseudodifferentiels à caracteristiques doubles, C. R. Acad. Sci. Paris Sér. A-B 275 (1972), A817-A819. 
6. J. Sjostrand, Parametrics for pseudodifferential operators with multiple characteristics (to appear).

7. F. Treves, Linear partial differential equations with constant coefficients: Existence, approximation and regularity of solutions, Math. and its Applications, vol. 6, Gordon and Breach, New York, 1966. MR 37 \#557.

8. - A link between solvability of pseudodifferential equations and uniqueness in the Cauchy problem, Amer. J. Math. 94 (1972), 267-288.

9. - Concatenations of second-order evolution equations applied to local solvability and hypoellipticity, Comm. Pure Appl. Math. 26 (1973), 201-250.

DEPARTMENT OF MATHEMATICS, RUTGERS UNIVERSITY, NEW BRUNSWICK, NEW JERSEY 08903 